\title{
IUE STUDIES OF LUMINOUS X-RAY BINARIES
}

\author{
J.B. Hutchings \\ Dominion Astrophysical Observatory \\ Herzberg Institute of Astrophysics \\ Victoria, B.C.
}

IUE has been used to study 11 high luminosity $X$-ray binaries, of which 3 are in the Magellanic Clouds. In the supergiant systems, X-ray ionisation bubbles have been found in most cases, leading to a greater understanding of the winds and accretion processes. Further studies of precessing objects such as LMC X-4 with IUE and ST are clearly of considerable interest, relating to $X$-ray heating and blanketing. Detailed studies of the Cyg $x-1$ ionisation bubble may resolve the long standing puzzle of its orbit inclination and masses. UV continua have furnished valuable information on extinction, temperatures and luminosities, and the presence of non-stellar (i.e. disk) luminosity. Here too, more detailed studies are clearly indicated for the future. A unique object of interest is the LMC transient 0538-66 whose UV spectrum has quasarlike lines and luminosity which varies oppositely to the visible. This may be a case of supercritical accretion generating an optically thick shell ("disk") about the pulsar.

IUE has provided important information on a unique galactic source, $0236+61$, which is also a radio variable and may be the only "young" $X$-ray neutron star. Its nature is still not understood, but IUE coverage of the 26-day cycle should contribute greatly to resolving the outstanding questions. Its UV spectrum shows a C IV wind (aspect selected?) in a rotating main sequence star, and several unusual line features.

Further time dependent studies with IUE can provide us with much more information on these important objects. IUE studies have also defined clearly the programs that need to be done with ST. This review will be published in full in the 1982 cospar proceedings.

Richard M. West (ed.), Highlights of Astronomy, Vol. 6, 648. Copyright 1983 by the $L A U$. 